OPEN ACCESS

Edited by:

Ralf J. Ludwig,

Universität zu Lübeck, Germany

Reviewed by:

Hiroshi Koga,

Kurume University School of

Medicine, Japan

Takashi Hashimoto,

Osaka City University, Japan

*Correspondence:

Sébastien Le Jan

sebastien.le-jan@univ-reims.fr

tThese authors have contributed equally to this work

Specialty section:

This article was submitted to Autoimmune and Autoinflammatory

Disorders,

a section of the journa

Frontiers in Immunology

Received: 25 June 2019

Accepted: 21 August 2019

Published: 11 September 2019

Citation:

Nesmond S, Muller C, Le Naour R, Viguier M, Bernard P, Antonicelli F and Le Jan S (2019) Characteristic Pattern of IL-17RA, IL-17RB, and IL-17RC in Monocytes/Macrophages and Mast

Cells From Patients With Bullous Pemphigoid.

Front. Immunol. 10:2107. doi: 10.3389/fimmu.2019.02107

\section{Characteristic Pattern of IL-17RA, IL-17RB, and IL-17RC in Monocytes/Macrophages and Mast Cells From Patients With Bullous Pemphigoid}

\author{
Stéphane Nesmond ${ }^{1}$, Céline Muller ${ }^{1}$, Richard Le Naour ${ }^{1,2}$, Manuelle Viguier $^{3}$, \\ Philippe Bernard ${ }^{1,3+}$, Frank Antonicelli ${ }^{1,4 \dagger}$ and Sébastien Le Jan ${ }^{1 * \dagger}$
}

${ }^{1}$ Laboratory of Dermatology, EA7509 IRMAIC, University of Reims-Champagne-Ardenne, Reims, France, ${ }^{2}$ Department of Biological Sciences, UFR Pharmacy, University of Reims Champagne-Ardenne, Reims, France, ${ }^{3}$ Department of Dermatology, University Hospital, University of Reims-Champagne-Ardenne, Reims, France, ${ }^{4}$ Department of Biological Sciences, Immunology, UFR Odontology, University of Reims-Champagne-Ardenne, Reims, France

Inflammation is largely implicated in bullous pemphigoid (BP), the most frequent skin auto-immune blistering disease. IL-17, essentially IL-17A/F, has been involved in blister formation through regulation of protease production, and its specific serum profile within $\mathrm{BP}$ was related to disease outcome. However, relationships between IL-17 family ligands and receptors are quite complex with six different $\mathrm{IL}-17$ isoforms, and five different receptors. We here aimed at clarifying the contribution of the $\mathrm{IL}-17$ axis in BP by characterizing not only the expression of IL-17 receptor (IL-17R) members within immune cells isolated from BP patients (PMNs, $n=9$; T-lymphocytes, $n=10$; and monocytes, $n=10)$ but also the expression of IL-17 isoforms in sera $(n=83)$, and blister fluid $(n=31)$ of BP patients. We showed that at diagnosis, IL-17RA and IL-17RC expression were significantly increased in monocytes isolated from BP patients as compared to those from control subjects ( $p=0.006$ and $p=0.016$, respectively). Notably, both IL-17RA and IL-17RC mRNA expression remained elevated in BP monocytes at time of relapse. We further demonstrated a significant increase of all IL-17 isoforms tested in BP blister fluid compared with BP serum (IL-17A, $p<0.0001$; IL-17A/F, $p<0.0001$; IL-17B, $p=0.0023 ;$ IL-17C, $p=0.0022 ;$ IL-17E, $p<0.0001$ ). Among all, IL-17B was the only cytokine for which a significant decreased concentration within blister fluid was observed in BP patients with severe disease compared to patients with moderate disease $(p=0.012)$. We further evidenced a significant negative correlation between IL-17B levels and blister/erosion BPDAl subscore $(r=-0.52, p=0.003)$. We finally identified mast cells as a potential target of IL-17B in lesional skin of BP patients. In conclusion, we showed here that IL-17RA and IL-17RC expression in monocyte was associated with disease activity and evidenced in situ a negative correlation between BP disease activity and IL-17B, whose effects could be mediated by IL-17RB expressed by mast cell in BP lesional skin.

Keywords: autoimmunity, inflammation, bullous pemphigoid, IL-17 receptors, IL-17 isoforms 


\section{INTRODUCTION}

Bullous pemphigoid (BP) is the most frequent blistering skin disease of autoimmune origin and affects mainly the elderly (1-6). Clinical features of BP are intense pruritus associated with tense bullae, inflammatory erythematous plaques, and itching $(1,2,6,7)$. BP is characterized by the production of autoantibodies against two proteins of the hemidesmosome structure, BP180, and BP230 (8-13). The binding of $\mathrm{BP}$ autoantibodies onto their target induces blister formation by activating complement pathway, which triggered subsequent recruitment of inflammatory cells at the dermalepidermal junction. Secretion of inflammatory key mediators by inflammatory cells leads up to an overexpression of proteases, such as the matrix metalloproteinase-9 (MMP-9) and the neutrophil elastase, which are involved in dermal-epidermal splitting (14-23). However, autoantibody level does not explain all clinical features of BP (24), suggesting that other regulatory mechanisms are involved in the inflammatory response associated with BP. In this line, we previously showed that IL-17 within the blister fluid (BF) of BP patients was related to MMP-9 production (25), and that specific patterns of IL-17 within the serum of BP patients were related to BP outcome (26). Moreover, anti-IL-17 therapy was revealed quite promising by using experimental murine and human-derived models of BP (27). However, these studies mostly concerned IL-17A and IL-17A/F isoforms, and to our knowledge, no study has evaluated the expression of the receptors of those cytokines yet, or the type of cells expressing those receptors in patients with BP.

IL-17 receptor family is composed of five members from IL17RA to IL-17RE. The subunit IL-17RA is ubiquitous (28-31) and is a common co-receptor subunit for other members of the IL-17 family. IL-17RA pairs with IL-17RC to bind either IL-17A or IL-17F as covalent homodimers, but also IL-17A/IL$17 \mathrm{~F}$ as heterodimers $(32,33)$. However, the association of IL17RA with other members of the IL-17 receptor family shifts the affinity toward other members of the IL-17 family (34). Indeed, combination of IL-17RA with IL-17RB binds IL-17E as covalent homodimers (35-37). Besides, IL-17RA can also associate with IL-17RE to bind IL-17C as homodimers (38). However, the involvement of IL-17RA is not compulsory, as IL-17RB forms homodimers to target IL-17B as covalent homodimers (39). Up to now, no ligand for IL-17RD has been discovered yet. Also, the receptor of IL-17D remains unknown. In BP, IL-17A production has been mainly attributed to neutrophils, $\mathrm{CD}^{+} \mathrm{T}$-lymphocytes, and mast cells $(25,27)$. Besides, IL-17RC expression was found increased in the skin of BP patients, whereas IL-17RA expression was unchanged (27). However, to better understand the role of IL-17 axis in BP, the pattern of IL-17 receptor isoform expression, and the cell types expressing those receptors both in the skin of BP patients and in the circulating immune blood cells need to be further investigated.

In this prospective study, we investigated the IL-17R isoform expression in immune blood cells of BP patients. We also analyzed the expression of IL-17 isoforms in the sera and in the $\mathrm{BF}$ of $\mathrm{BP}$ patients. To investigate the clinical involvement of the different IL-17 members in BP, we performed correlation studies between IL-17 concentration within the BF and the BPDAI (bullous pemphigoid disease activity index). Finally, to further delineate the role of IL-17 members in the inflammatory response within the BF associated with clinical activity of BP, we evaluated the expression of the respective IL-17 receptors at the surface of immune cells in lesional skin of patients.

\section{MATERIALS AND METHODS}

\section{Patients and Study Design}

This prospective, single-center study was conducted between September 2013 and July 2017 in the Department of Dermatology at Reims University Hospital (French Referral Center for Autoimmune Bullous Diseases), under the approval of the Ethic Committee of the University Hospital of Reims (CNIL authorization DR-2013-320). In accordance with the Helsinki Declaration, all subjects gave their informed and written consent before their inclusion in the study. Patients that were included presented blistering skin dermatosis fulfilling at least three of four clinical criteria for BP according to Vaillant et al. (40), and in lesional skin, a basal lamina continuous deposit of IgG and/or C3 is revealed by direct immunofluorescence (IF) microscopy. Exclusion criteria were administration of a specific treatment for more than 2 days, pregnancy, and expected survival shorter than 3 months. Sera and blood cells were collected at different time points: at diagnosis (D0), 360 days after the diagnosis (D360), and at the time of a relapse (if it occurred). When possible, BF was collected at diagnosis (D0). Control samples were obtained from age- and sex-matched patients admitted to the department of traumatology, and orthopedic surgery of the same hospital. Controls that were included in the study did not have any autoimmune diseases and any clinical or biological signs of inflammation.

\section{Clinical Characteristics of BP Patients}

Clinical data recorded at baseline were gender, age, and clinical activity of the disease evaluated by the Bullous Pemphigoid Disease Area Index (BPDAI) score (7). The BPDAI measures separate scores for mucous membrane and skin activities, the latter evaluating separately both cutaneous urticaria/erythema (non-bullous phase), and cutaneous blisters/erosions (blistering phase). As previously reported (41), a global BPDAI score $\geq 56$ at baseline defined a severe disease while a global BPDAI score $<56$ defined a moderate disease.

\section{Cell Isolation}

Peripheral blood mononuclear cells (PBMCs) and polymorphonuclear cells (PMNs) were isolated by densitygradient centrifugation from EDTA-treated whole blood (Granulosep, Eurobio-Abcys, France). Monocytes were then purified from PBMCs by means of positive selection with CD14 immunomagnetic beads (MACS; Miltenyi Biotec, Germany), according to the manufacturer's instructions. Then, flow through was used to isolate $\mathrm{T}$ lymphocytes by means of negative selection with the pan T-cell Isolation Kit from Miltenyi Biotec (MACS). 


\section{IL-17R Gene Expression Analysis}

Total RNA was extracted from isolated cells (3M, 5M, 7M, and $4 \mathrm{M}$ for monocytes, lymphocytes, PMNs, and PBMCs, respectively) using TRI-Reagent (Euromedex, Bas-Rhin, France) according to the manufacturer's protocol. cDNAs were synthetized and amplified using the Maxima First Strand cDNA kit with dsDNAse (Life Technologies), according to the manufacturer's instructions. The expression of IL-17RA, IL-17RB, IL-17RC, IL-17RD, IL-17RE, and $\beta 2$-microglobulin $\left(\beta_{2} \mathrm{M}\right)$ was analyzed by real-time quantitative PCR using the Power SYBR Green PCR Master Mix (Applied Biosystems) on the Stratagene Mx3005P (Agilent Technologies). Relative quantification was performed with $\beta_{2} \mathrm{M}$ as a reference gene. Results were analyzed by using the MxPro QPCR software, and the fold regulation was calculated by using the $2^{-\Delta \Delta C T}$ method. Primer sequences (Eurofins) used to detect IL-17RA, IL-17RB, IL-17RC, IL-17RD, IL-17RE, and $\beta_{2} \mathrm{M}$ are detailed in Table 1.

\section{Cytokine Measurement in Biological Fluids From BP Patients}

IL-17A, IL-17A/F, IL-17B, IL-17C, and IL-17E were measured in control sera $(n=46)$, BP sera $(n=83)$, and BP BF $(n=$ 31) using a U-PLEX assay (MesoScale Diagnostics; Rockville; USA). U-PLEX technology allows multiplex measurement of up to 10 cytokines within a single well in a volume of 50 $\mu \mathrm{L}$. This technique is based on electro-chemiluminescence detection. Briefly biotinylated capture antibodies were coupled to U-PLEX linkers. The U-PLEX linkers then self-assembled onto unique spots on the U-PLEX plate. After binding to the capture antibodies, detection antibodies conjugated with electrochemiluminescent labels (MSD GOLD SULFO-TAG) bound to the analytes to complete the sandwich immunoassay. The plate was then placed into an MSD instrument (SECTOR S6000 plate reader) to acquire data. Data analysis was performed by using MSD Workbench software. Limits of detection (LLOD) were 1.6 $\mathrm{pg} / \mathrm{ml}$ for IL-17A, $3.0 \mathrm{pg} / \mathrm{ml}$ for IL-17A/F, $1.0 \mathrm{pg} / \mathrm{ml}$ for IL-17B, $3.0 \mathrm{pg} / \mathrm{ml}$ for IL-17C, and $0.76 \mathrm{pg} / \mathrm{ml}$ for IL-17E.

\section{IL-17R Detection in Isolated Monocytes From BP Patients}

IL-17RA and IL-17RC expression was analyzed in monocytes isolated from BP patients and control subjects by immunocytochemistry (ICC). Isolated monocytes were cytospun and fixed with paraformaldehyde 4\% (VWR). The primary

TABLE 1 | Primer sequences used for amplification of IL-17R member and $\beta 2 \mathrm{M}$ genes by real-time quantitative PCR.

\begin{tabular}{lll}
\hline Oligonucleotide & Forward sequence & Reverse sequence \\
\hline IL-17RA & TGCCCCTGTGGGTGTACTGGT & GCAGGCAGGCCATCGGTGTA \\
IL-17RB & TACCCCGAGAGCCGACCGTT & GGCATCTGCCCGGAGTACCCA \\
IL-17RC & CTGCCCTTGTGCAGTTGG & CAGATTCGTACCTCACTCCCTA \\
IL-17RD & AGGCCTGGGTGAGGAGGAACC GGGGAATCAGAGGGAGGCAGCA \\
IL-17RE & CCACCTTCAGGCCATGCAGCC & CTGTCATCCGTGTGGGAGGCC \\
$\beta_{2} \mathrm{M}$ & ACCCCCACTGAAAAGATGA & ATCTTCAAACCTCCATGATG
\end{tabular}

antibodies rabbit anti-human IL-17RA (Bioss Antibodies, bs-2606R), and rabbit anti-human IL-17RC (Bioss Antibodies, bs-2607R) were applied to the cells and incubated overnight at $4^{\circ} \mathrm{C}$. Chicken anti-rabbit IgG Alexa Fluor 594 (Life technologies, A21442) was used as secondary antibodies. Nuclei were stained with Hoechst 33342 (Thermofisher, Waltham, MA).

\section{IL-17R Detection in BP Skin Biopsy Specimen}

Double IF staining were performed to analyze IL-17RA, IL17RB, and IL-17RC expression on paraformaldehyde-fixed and paraffin-embedded lesional skin biopsy specimens from three BP patients before introduction of any treatment. All biopsies were obtained from the Pathology Department of Reims University Hospital. Briefly, after heat-induced antigen retrieval in Tris $10 \mathrm{mM}$ EDTA buffer $\mathrm{pH} 9$ and blocking in PBS $1 \times /$ BSA $3 \%$, the primary antibodies rabbit anti-human IL-17RA (Bioss Antibodies, bs-2606R), rabbit anti-human IL-17RB (Gene Tex, GTX81729), rabbit anti-IL-17RC (Bioss Antibodies, bs2607R), mouse anti-human mast cell tryptase clone AA1 (Dako,

TABLE 2 | Baseline clinical characteristics of patients with BP.

(A) In the whole BP population included $(n=83)$

\begin{tabular}{ll}
\hline Number of daily new blisters $^{*}$ & $21 \pm 36$ \\
BPDAl total score* $^{\star}$ & $40 \pm 27$ \\
BPDAl skin activity score* $^{\star}$ & $38 \pm 26$ \\
- Blisters/erosions score $^{*}$ & $25 \pm 17$ \\
- Erythema/urticaria score* $^{\star}$ & $14 \pm 14$ \\
Patients with severe disease $^{\mathrm{a}}, n(\%)$ & $22(26)$ \\
Patients with relapse $^{\mathrm{b}}, n(\%)$ & $12(14)$
\end{tabular}

(B) In the subgroups of BP patients with $(n=12)$ and without relapse $(n=71)$

\begin{tabular}{lccc}
\hline & $\begin{array}{c}\text { BP without } \\
\text { relapse }\end{array}$ & $\begin{array}{c}\text { BP with } \\
\text { relapse }\end{array}$ & $\boldsymbol{p}$-value \\
Number of daily new blisters $^{*}$ & $21 \pm 36$ & $22 \pm 32$ & 0.2 \\
BPDAl total score $^{\star}$ & $38 \pm 27$ & $49 \pm 25$ & 0.1 \\
BPDAl skin activity score $^{\star}$ & $37 \pm 27$ & $47 \pm 25$ & 0.2 \\
- Blisters/erosions score $^{\star}$ & $24 \pm 17$ & $32 \pm 21$ & 0.2 \\
- Erythema/urticaria score $^{*}$ & $14 \pm 15$ & $15 \pm 12$ & 0.5 \\
\hline
\end{tabular}

(C) In the subsets of BP patients with moderate $(n=61)$ and with severe disease $(n=22)$

Number of daily new blisters* BPDAl total score*

BPDAl skin activity score* - Blisters/erosions score* - Erythema/urticaria score*

$\begin{array}{ccc}\text { Moderate BP } & \text { Severe BP } & \boldsymbol{p} \text {-value } \\ 9 \pm 13 & 57 \pm 52 & 0.0001 \\ 26 \pm 15 & 77 \pm 17 & 0.0001 \\ 25 \pm 15 & 75 \pm 15 & 0.0001 \\ 17 \pm 11 & 47 \pm 13 & 0.0001 \\ 9 \pm 10 & 28 \pm 16 & 0.0001\end{array}$

a Severe disease was defined by BPDAl $\geq 56$ (41).

${ }^{b}$ Relapse was defined as the reappearance of at least three daily new blisters along with pruriginous, erythematous, or urticarial plaques.

Mann-Whitney's test was used for statistical analysis.

*Mean \pm SD. 
M7052), mouse anti-human CD163 (Novusbio, NB110-59935), and mouse anti-myeloperoxidase FITC (Abcam, ab11729) were applied and incubated overnight $4{ }^{\circ} \mathrm{C}$. Goat anti-mouse IgG Alexa Fluor 488 (Life technologies, A11029) and chicken anti-rabbit IgG Alexa Fluor 594 (Life Technologies, A21442) were used as secondary antibodies. Nuclei were stained with Hoechst 33342 (Thermofisher, Waltham, MA).

ICC and IF staining were visualized on an AxioObserver Z1 microscope (ZEISS) spinning disk ILAS 2 (Roper scientific). Image analysis was performed by using Metamorph software (Roper Scientific).

\section{Statistical Analysis}

Statistical significance was inferred when necessary. GraphPad Prism 5 software (GraphPad, La Jolla, CA) was used for statistical analysis. Results are presented as mean \pm SEM (standard error of the mean). Normality test was performed to evaluate Gaussian's distribution of the different population tested. We used nonparametric Mann-Whitney's test to compare two independent groups and non-parametric Wilcoxon's test to compare paired groups of population; chi-square test was used to evaluate qualitative variables. Pearson's correlation test was performed to explore the relationship between continuous variables. Results were considered significant when $p<0.05$.

\section{RESULTS}

\section{Characteristics of the Studied Population}

A total of 83 patients with BP were included in the study. The mean age at diagnosis was 81 years old and the sex ratio $\mathrm{F} / \mathrm{M}$ was 1.9. A population of control subjects $(n=46)$ was used with no significant difference regarding these factors. At diagnosis, the total BPDAI score in the whole BP population was $40 \pm 27$ (Table 2). BP patients received superpotent topical corticosteroids as treatment, in association or not with systemic immunomodulators. Among the 83 patients included, 61 patients had a moderate BP, 22 patients a severe BP, 12 (14\%) relapsed, 19 patients $(24 \%)$ died, and $6(7 \%)$ were lost during the year of follow-up. Baseline clinical characteristics of BP patient subgroups according to outcome are displayed in Table 2.

\section{IL-17 Receptor Family Members in Immune Circulating Cells in BP}

We first investigated the expression of each IL-17 receptor family member in circulating immune cells of patients with BP. At the time of diagnosis, circulating immune cells issued from BP patients or control subjects showed differential expression profile of each IL-17 receptor member. Indeed, whereas PMN cells mainly expressed IL-17RA mRNA (Figure 1A), lymphocytes expressed all IL-17 receptor members, except IL-17RD (data not shown), both in patients with BP and in control subjects (Figure 1B). In addition, in both lymphocytes and PMNs, no statistical differences could be observed at diagnosis between cells originated from BP patients and cells issued from control subjects. In contrast, a significant increase of IL-17RA, and IL17RC mRNA expression ( $p=0.006$ and $p=0.016$, respectively) was evidenced in monocytes from BP patients $(n=10)$ as compared with control subjects $(n=6)$ (Figure 1C). The overexpression of both receptors in monocytes from BP patients was also demonstrated at the protein level by IF staining (Figure 1D). Evaluation of the longitudinal variation of these receptors was realized on BP patients with an ongoing remission $(n=7)$, and in BP patients with relapse $(n=5)$ for whom blood samples were available. Baseline clinical characteristics in these subgroups of BP patient with and without relapse were similar to those displayed in Table 2 (data not shown). Both IL-17RA and IL-17RC mRNA increased expressions were significantly abolished after 1 year of treatment (D360) as compared to the level detected at the time of diagnosis (D0) ( $p=0.016$ and $p$ $=0.016$, respectively) in monocytes from patients with ongoing remission (Figure 2A). In contrast, in patients with relapse, both IL-17RA, and IL-17RC mRNA expression remained elevated at time of relapse ( $\left.D_{\text {Relapse }}\right)$. Indeed, no significant decrease of both IL-17RA and IL-17RC was demonstrated at time of relapse compared with diagnosis (Figure 2B).

\section{IL-17 Isoforms in Serum and BF of Patients With BP}

We next examined the expression of the IL-17 ligands including IL-17A, IL-17A/F, IL-17B, IL-17C, and IL-17E in biological fluids that could bind to the above-described IL-17R members expressed by circulating immune cells originated from BP patients. At the time of diagnosis, all tested IL-17 isoforms were detected in sera of control subjects $(n=46)$, and in sera $(n=83)$, and BF $(n=31)$ of patients with BP (Figure 3$)$. Whatever the IL17 isoforms analyzed, no significant differences were evidenced between sera from BP patients and sera from control subjects. In contrast, in BF of BP patients, the mean levels of each IL-17 isoform were all significantly increased as compared to BP sera concentrations (IL-17A, $p<0.0001$; IL-17A/F, $p<0.0001$; IL$17 \mathrm{~B}, p<0.01$; IL-17C, $p<0.01$; IL-17E, $p<0.0001$ ) (Figure 3).

\section{IL-17 Isoforms and Disease Activity}

Investigation of whether the IL-17 isoform levels within the BF varied according to disease activity at diagnosis was performed on $16 \mathrm{BP}$ patients with "mild to moderate" disease and on 15 BP patients with "severe" disease (Figure 4A). These subgroups were characterized by an increase of the total BPDAI score and skin subscores in BP patients with severe disease (BPDAI total score: $72 \pm 14$ vs. $39 \pm 13, p=0.0001$; BPDAI skin activity subscore: $71 \pm 14$ vs. $38 \pm 13, p=0.0001$; blisters/erosions subscore: $43 \pm 11$ vs. $26 \pm 9, p=0.0003$; erythema/urticaria subscore: $29 \pm 16$ vs. $12 \pm 10, p=0.003$ for severe and moderate BP patients, respectively). Figure 4A showed that IL$17 \mathrm{~B}$ level was significantly higher ( 3 -fold, $p=0.01)$ in BF of BP patients with a mild to moderate disease as compared with levels measured in BF of BP patients with a severe disease. None of the other IL-17 isoforms showed significant variations according to disease extent. Deeper investigation revealed a significant and negative correlation between IL-17B and total BPDAI score ( $n$ $=31, r=-0.51, p=0.004)$. Further analysis with respect to BPDAI subscores showed that IL-17B concentrations in BF were negatively correlated with the total skin BPDAI subscore ( $n=31, r=-0.51, p=0.003)$, and the skin blisters/erosions 
A

PMNs
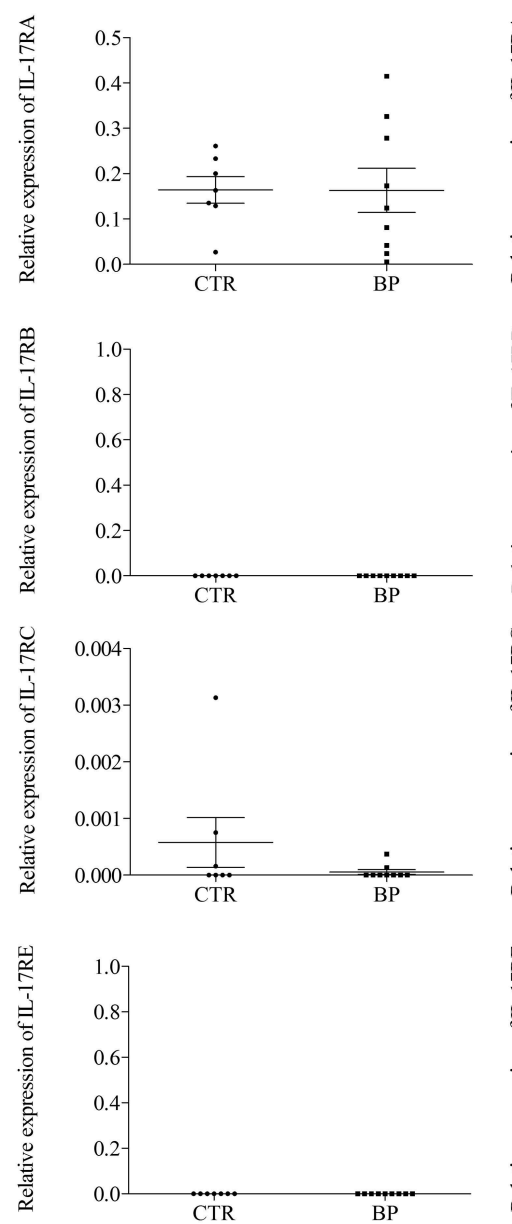

B

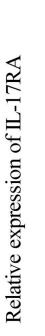
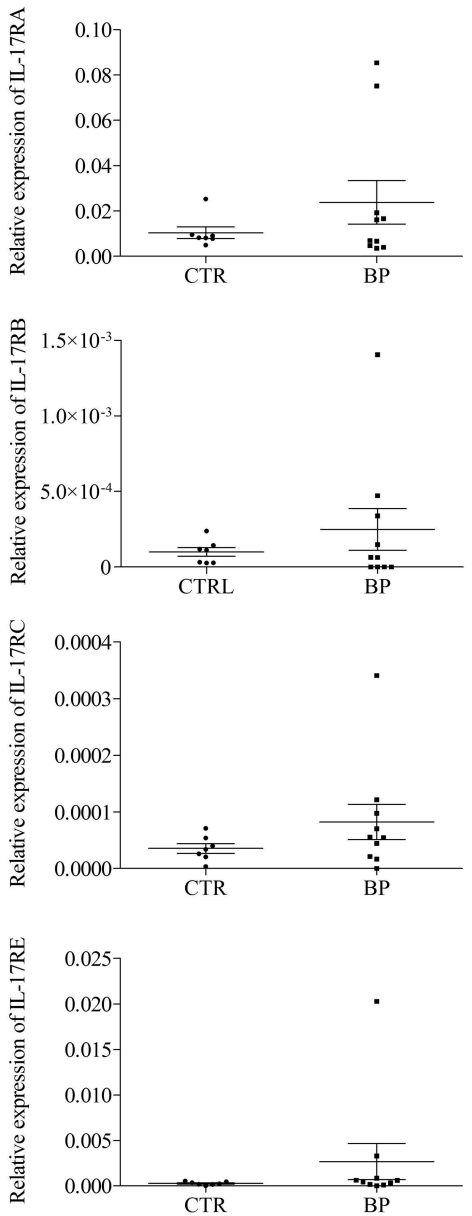

D

IL-17RA

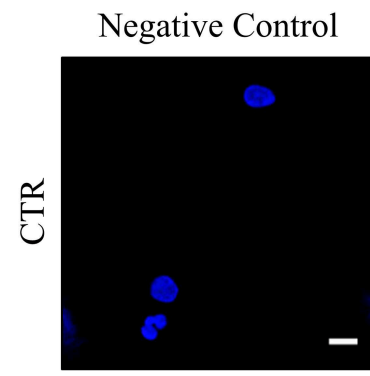

C
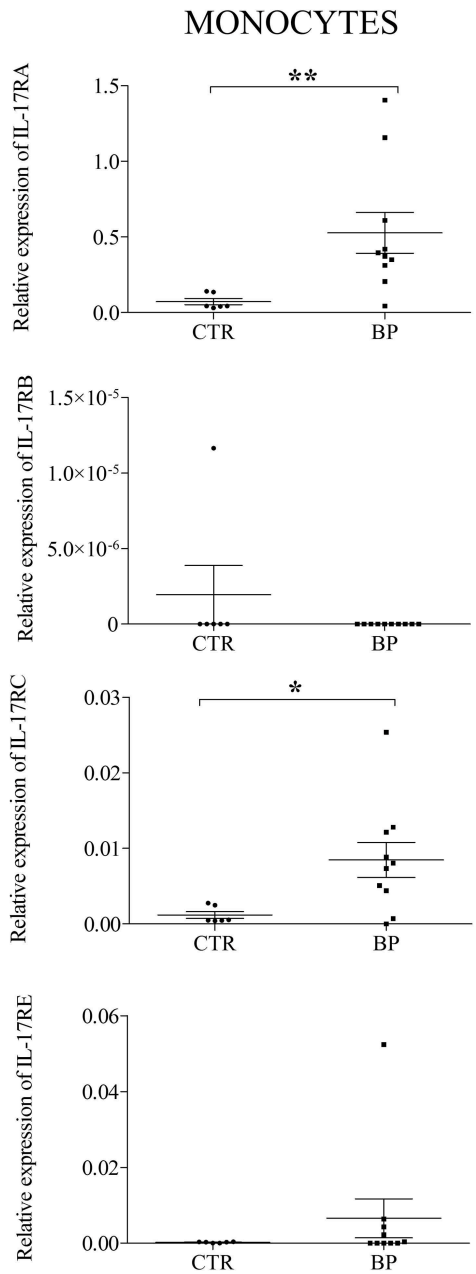

\section{IL-17RC}
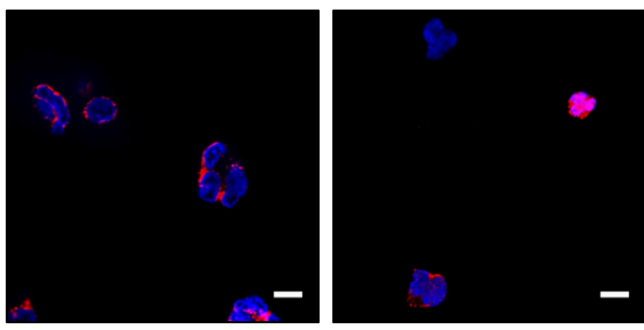

6

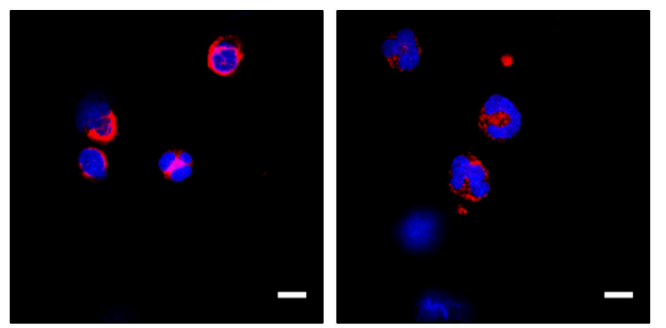

FIGURE 1 | IL-17RA and IL-17RC mRNA expressions were increased in monocytes isolated from blood of patients with BP. The mRNA expression of IL-17RA, IL-17RB, IL-17RC, and IL-17RE were analyzed by real-time quantitative PCR in polymorphonuclear cells (PMNs) (A), in lymphocytes (B), and in monocytes

(Continued) 
FIGURE 1 | (C) isolated from blood of control subjects (CTR), and of patients with BP at diagnosis (BP). Lines represent the mean, and non-parametric Mann-Whitney's test was used for statistical analysis ( ${ }^{*} p<0.05 ;{ }^{* *} p<0.01$ ). (D) Monocytes from BP patients and control subjects were stained for IL-17RA or IL-17RC. Negative control: primary antibodies were not added. Nuclei were counterstained with Hoechst (blue). Scale bar $=10 \mu \mathrm{m}$.
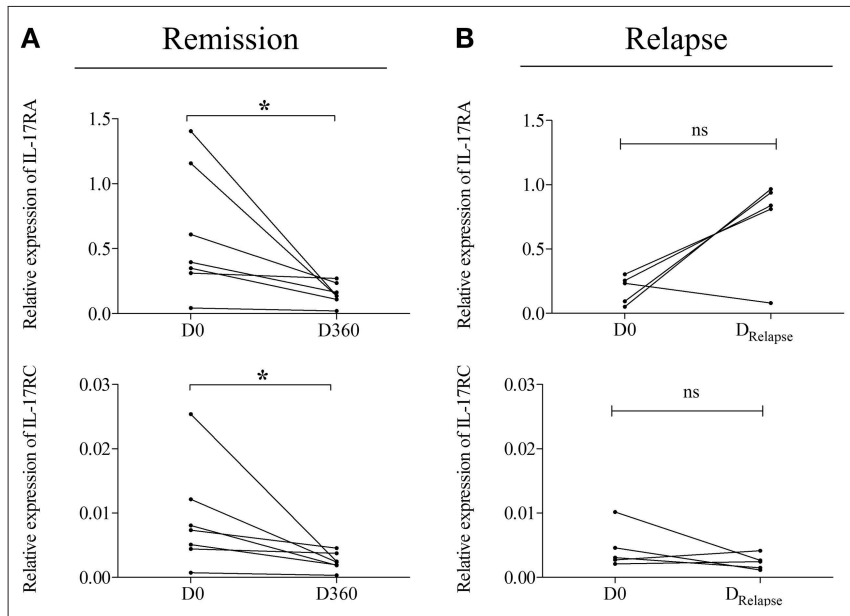

FIGURE 2 | Analysis of IL-17RA and IL-17RC expression in monocytes issued from patients with BP according to disease outcome. IL-17RA and IL-17RC mRNA expressions were analyzed in monocytes of BP patients with ongoing remission $(\mathbf{A})$ and of $B P$ patients who relapsed $(\mathbf{B})$, at diagnosis $\left(D_{0}\right)$, and at day $360\left(D_{360}\right)$, or at the time of relapse (DRelapse), respectively. Non-parametric paired Wilcoxon's test was used for statistical analysis ( ${ }^{\star} p<0.05 ; \mathrm{ns}=$ non-significant).

BPDAI subscore $(n=31, r=-0.52, p=0.003)$ but not with the erythema/urticaria BPDAI subscore $(n=31, r=-0.27$, $p=0.14)$ (Figure 4B).

\section{IL-17 Receptor Expression in Lesional Skin of BP Patients}

Finally, we wondered which cells within the blister cavity could be targeted by IL-17B. Double staining for IL-17RB and either mast cell (tryptase), macrophage (CD163), or neutrophil (myeloperoxidase, MPO) markers was performed in biopsies of BP patients $(n=3)$. Double staining revealed co-localization between IL-17RB and mast cell marker. Conversely, IL-17RB was not expressed by macrophages or neutrophils in blister cavity. In contrast, IL-17RA, and IL-17RC were expressed by both CD163 ${ }^{+}$ macrophages and $\mathrm{MPO}^{+}$neutrophils in blister cavity (Figure 5).

\section{DISCUSSION}

In this study, we demonstrated that IL-17 R expression displayed a peculiar pattern, with IL-17RA, and IL-17RC increased expression in monocyte and macrophages, whereas IL-17 RB was detected in mast cells. Noteworthy, IL-17RA and IL-17RC expression remained elevated in $\mathrm{BP}$ patients who relapsed under corticosteroid treatment. Furthermore, we also found that IL$17 \mathrm{~A}, \mathrm{~B} \mathrm{C}, \mathrm{E}$, and $\mathrm{F}$ isoforms were all increased in the $\mathrm{BF}$ of BP patients. Although such IL-17 production suggested a broad involvement of the IL-17 axis in BP with active skin lesions before starting treatment, we only evidenced a negative correlation between BP disease activity and IL-17B, which could be mediated by IL-17RB expressed by mast cell in BP lesional skin.

We also showed that at the active phase of the disease and before treatment, IL-17RA and IL-17RC expressions were increased in monocyte, but neither in lymphocytes nor in neutrophils. Furthermore, no variation was observed in PBMCs (data not shown), suggesting that the overexpression is mainly restricted to monocytes. Actually, the critical role of monocytes in BP was already illustrated in a previous study showing that monocytes but not lymphocytes from BP patients responded to CXCL10 by increasing MMP-9 secretion (42). Thus, although all IL-17 receptors were detected in circulating blood cells, the overexpression of IL-17RA and IL-17RC in monocytes suggests that those receptors could play a critical role in $\mathrm{BP}$. Actually, IL-17RA, and IL-17RC overexpression in monocytes could participate in BP relapse mechanisms as we observed that their expression was downregulated in BP patients whose disease was controlled by treatment, but remained overexpressed in BP patients who relapsed. Such results are in line with the IL-17 overexpression in the serum of BP patients with relapse under treatment (26) and with the capacity of those sera to increase NETosis in BP (43). This is also in setting with the fact that IL-17RC expression was found increased in the skin of BP patients (27), although in this study the authors found that IL-17RA expression remained unchanged. Indeed, in relation with the variations in monocytes, we also observed that IL-17RA and IL-17RC were markedly expressed by macrophages in the lesional skin of BP patients. Noteworthy, such IL-17RA, and IL-17RC expression in blister cavity was also demonstrated on neutrophils, further advocating on the role of both macrophages, and neutrophils in the dermal-epidermal cleavage upon IL-17 stimulation $(25-27,42,43)$. Therefore, altogether, our results showed that investigation of both IL-17 isoforms and IL17RA/RC may represent adequate tools to predict BP activity and outcome.

Here, we evidenced for the first time the expression of the different isoforms of IL-17 in the serum and in the BF of patients with BP. Furthermore, all of the IL-17 isoforms were produced at a higher level in the $\mathrm{BF}$ as compared to the serum of BP patients, suggesting a local production of these cytokines within the bullous area. However, the different IL-17 isoforms were produced at different levels. This is of importance as we also demonstrated that the expression of IL-17 receptors varied according to the immune cell types analyzed. Thus, this suggests that in BP, all combination of IL-17/IL-17R could be formed depending on the receptor types expressed at the surface of the cells, and on the affinity between these couples. IL-17A expression has already been mainly attributed to neutrophils, $\mathrm{CD}^{+}$T-lymphocytes, and mast cells $(25,27)$. The expression 

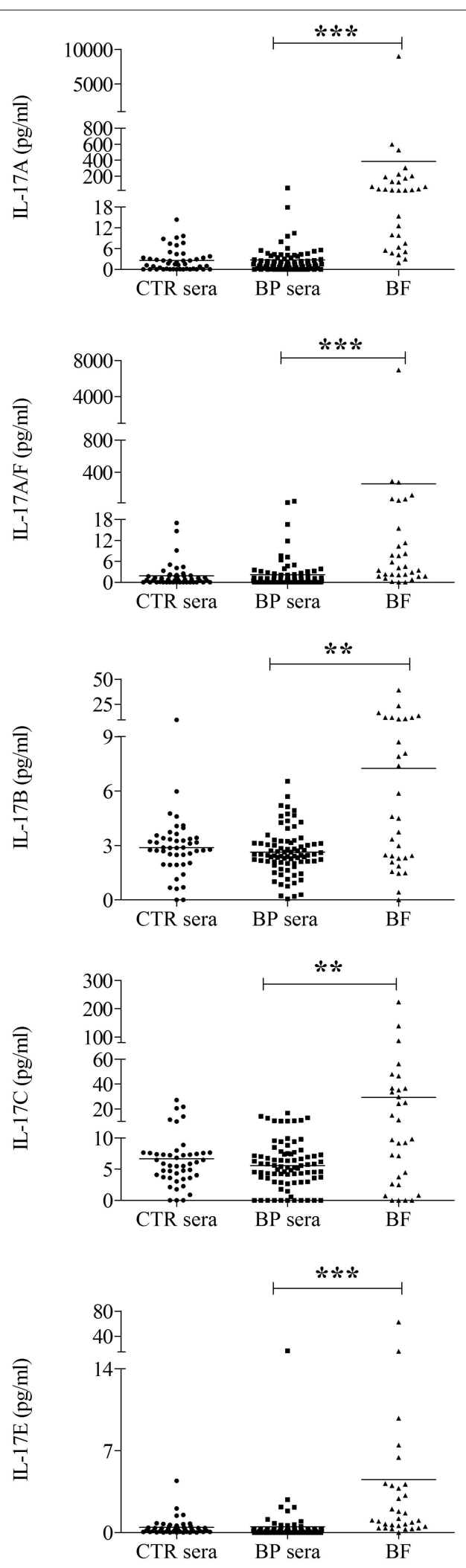

FIGURE 3 | The expression of each IL-17 isoform tested was increased in blister fluid of BP patients. The concentrations of IL-17A, IL-17A/F, IL-17B,

(Continued)
FIGURE 3 | IL-17C, and IL-17E were measured at the time of diagnosis in serum (BP sera), and blister fluid (BF) of BP patients, and in serum of control subjects (CTR sera) using U-PLEX cytokine multiplex assay. Lines represent the mean, and non-parametric Mann-Whitney's test was used for statistical analysis $\left.{ }^{* *} p<0.01 ;{ }^{* * *} p<0.001\right)$.

of other isoforms of IL-17 is much less defined. The expression of IL-17B and IL-17C has been attributed to T-cells (44). The expression of IL-17E has also been found to be produced by immune cells such as macrophage, lymphocyte, or mast cell $(37,45)$. Although the origin of the different IL-17 isoforms and of their receptors still needs to be further documented, the expression of all of those molecules of the IL-17 axis might conduct to a very intricate network in BP.

At baseline, only IL-17B expression was correlated with the BPDAI scores. Thus, we evidenced here, for the first time in $\mathrm{BP}$, an IL-17 cytokine isoform capable of discriminating BP patients according to disease activity with the particularity that a negative correlation was observed between IL-17B and BP activity. Although further studies are required to demonstrate whether IL-17B expression is only a marker of disease activity or if this cytokine has a specific role in the regulation of the inflammatory response in $\mathrm{BP}$, previous observation supports the idea that IL-17B could have a protective role in the process of inflammation (46). In BP, BPDAI subscore analysis showed that IL-17B was negatively correlated with the skin blisters/erosions lesions but not with the erythema/urticaria lesions. Therefore, IL-17B might not be involved in the pre-bullous phase of the disease. In contrast, the negative correlation between IL-17B and blisters and erosions lesions suggests that a BP patient with high IL-17B level would not form many blisters and erosions, and vice versa. IL-17B could interfere on $\mathrm{BP}$ pathogenesis by several mechanisms. On one hand, IL-17B could bind to IL-17RB, and scavenger at least in part IL-17RA to form the heterodimer IL17RA/IL-17RB. This would reduce the pool of IL-17RA to enable to transduce the pathogenic effects of IL-17A and IL-17F either as homodimers or as heterodimers. In this way, this could limit the effects of IL-17E (IL-25) that bind to the IL-17RA/RB homodimer (46). On the other hand, binding of IL-17B onto its receptor could also transduce an anti-inflammatory response. Indeed, it has been shown that IL-17B is an anti-inflammatory cytokine (46). Given that IL-17RB is expressed by mucosal epithelial cells, such effects could be involved in the regulation of mucosal inflammation that occurs in certain patients with BP (47). However, why a patient would express a higher IL-17B than another patient is still unknown, and whether this level varies according to disease extent for a specific patient still needs to be investigated.

Besides, IL-17B concentrations within the BF were correlated neither with the anti-BP180NC16A nor with the anti-BP230 autoantibody titers (data not shown), suggesting that, in BP, IL-17B production was disconnected from the autoimmune process, which initiates blister formation. Also, no correlation was observed between serum and BF concentrations (data not shown), suggesting that IL-17B is mainly produced at the skin lesional site. Although we mentioned the pathogenic role of IL$17 \mathrm{~A}$ or IL-17A/F above, we did not find any correlation with 


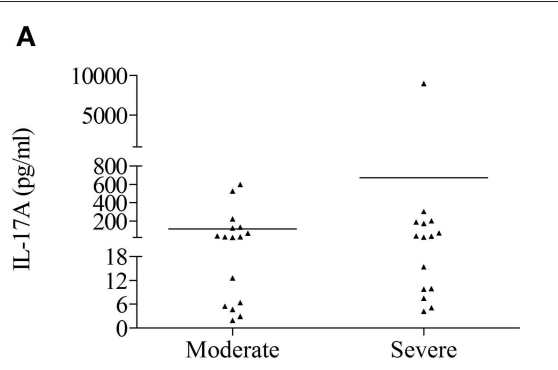

B
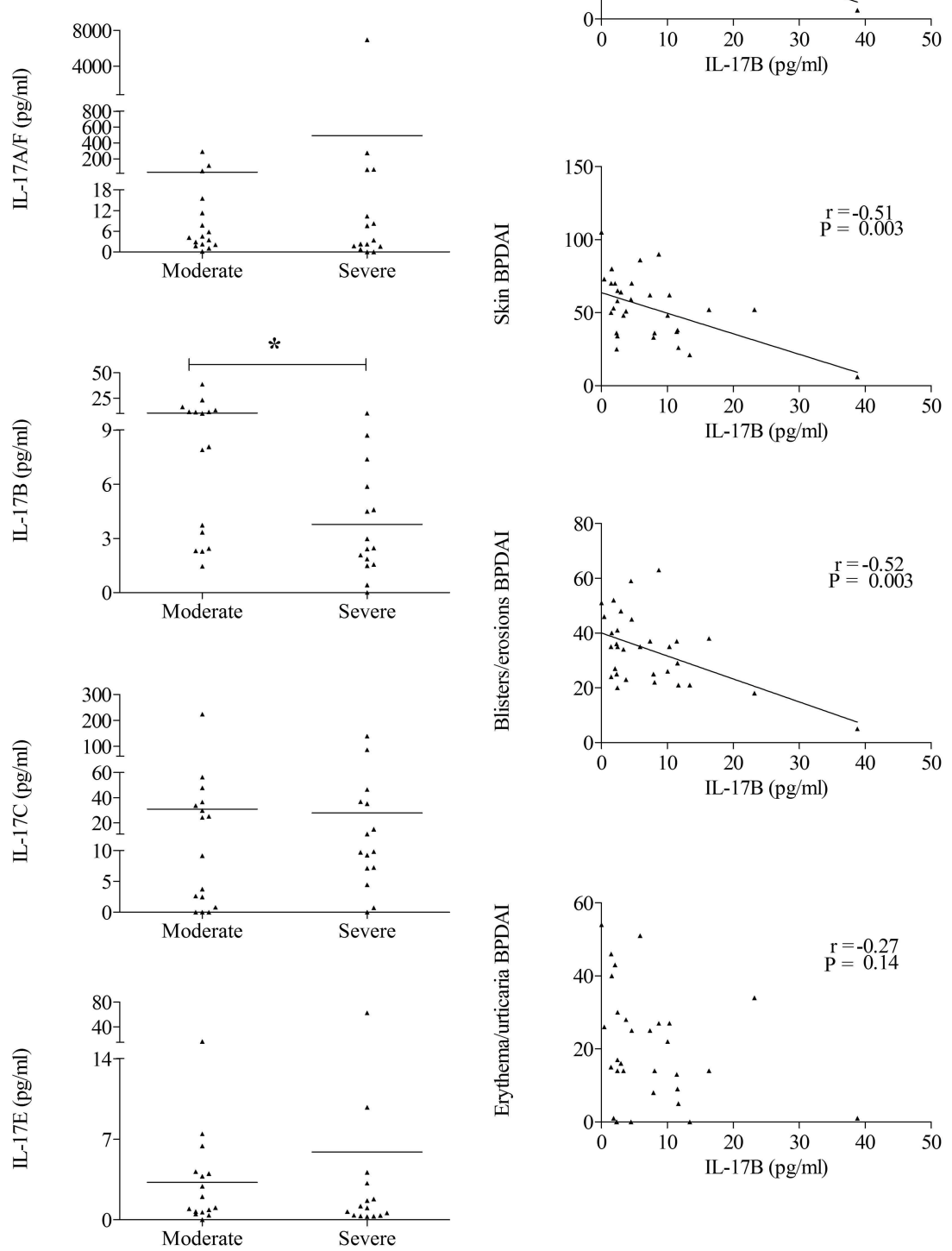

FIGURE 4 | IL-17B levels were decreased in BP patients with severe disease extent and negatively correlated with blisters/erosions BPDAI subscore. (A) IL-17A, IL-17A/F, IL-17B, IL-17C, and IL-17E concentrations measured at the time of diagnosis in BF were analyzed according to the disease extent. "Moderate" stands for BP patients with a BPDAI $<56$ and "Severe" for BP patients with a BPDAI $\geq 56$. Non-parametric Mann-Whitney's test was used for statistical analysis ( ${ }^{*} p<0.05$ ).

(B) Correlations between total, skin, blisters/erosions, or erythema/urticaria BPDAl scores, and IL-17B levels in BF were analyzed using the Pearson's correlation test.

IL-17A or IL-17A/F with the BPDAI score and subscores. A potential explanation could be that the binding of an IL-17 isoform onto an IL-17 receptor may modulate the affinity and specificity of other IL-17 isoform receptor-binding event. This is of interest as this suggests that besides the IL-17RA pathways, other IL-17/IL-17R signaling cascades may be involved in the pathophysiology of BP. Noteworthy, IL-17RB also associates with IL-17RA to transduce the effects of IL-17E. In our study, we 
Tryptase
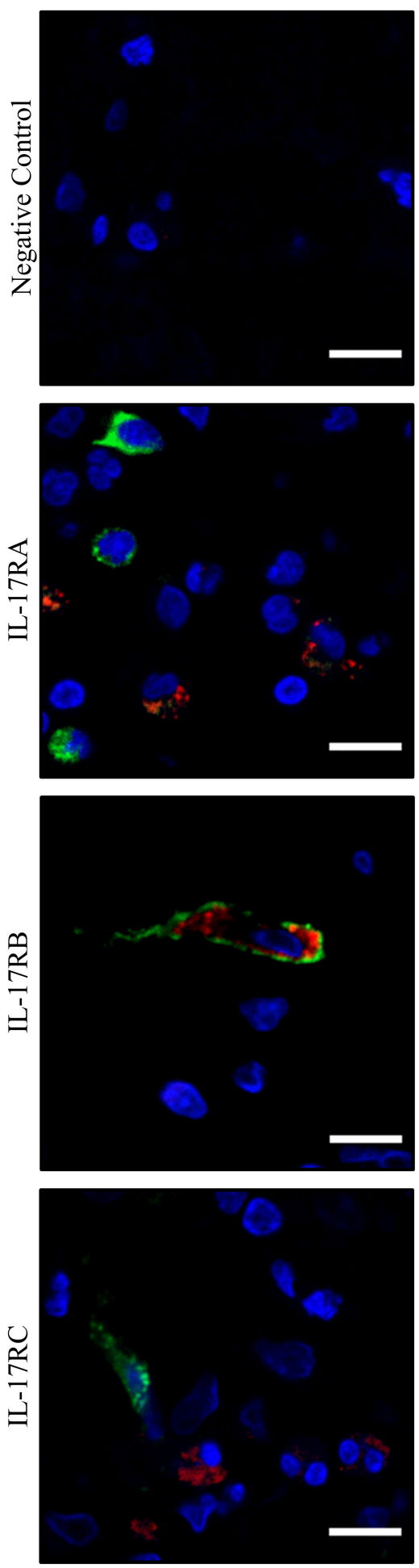

CD163
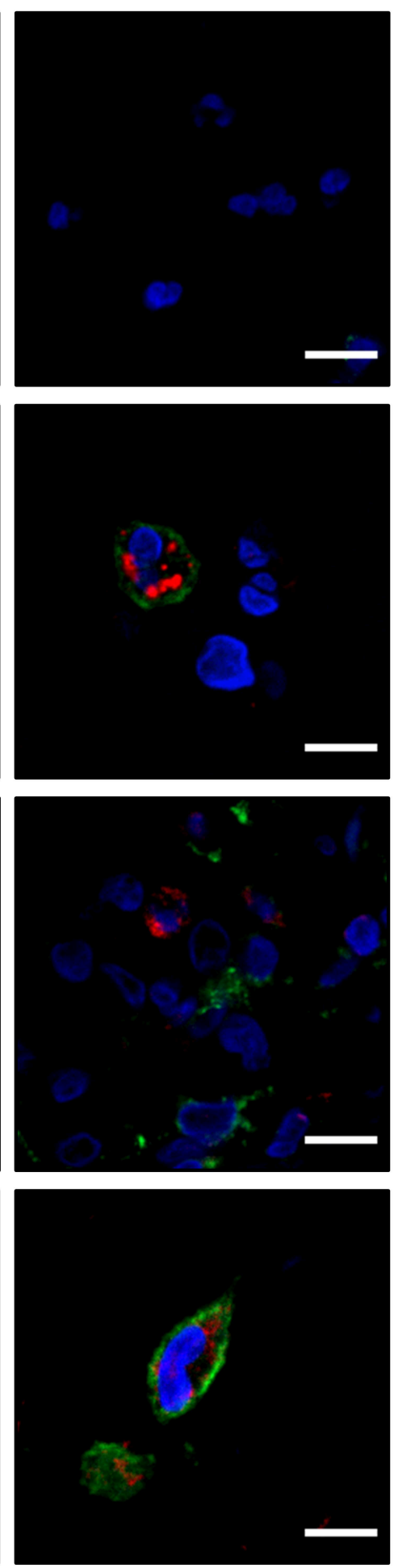

Myeloperoxidase
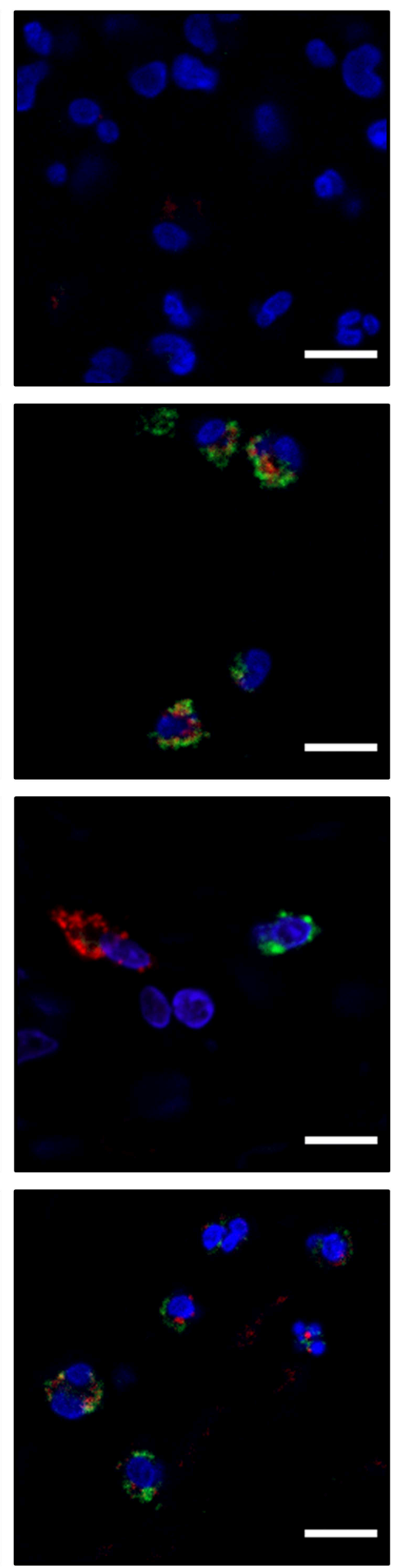

FIGURE 5 | IL-17RA, IL-17RB, and IL-17RC showed differential pattern of expression in immune cells associated to lesional skin of patients with BP. Paraffin-embedded skin biopsy specimen of blistering skin from BP patients $(n=3)$ were subjected to double immunofluorescence staining for IL-17RA, IL-17RB, and IL-17RC (in red), and either Tryptase, CD163, or myeloperoxidase (MPO) (in green) with Hoechst counterstain. Negative control: primary antibodies were not added. Scale bar $=10 \mu \mathrm{m}$. 
evidenced that the in situ expression of IL-17RB was attributed to mast cell within the blister cavity. This is in line with a previous study showing that mast cells expressed and responded to IL-17E, which also has IL-17RB as receptor (37). Thus, altogether, our results suggest that antagonist mechanisms may be involved in the formation of different IL-17 cytokine, and receptor complexes. However, further studies are required to better understand how the IL-17 network is related with clinical disease activity in BP.

Regarding the IL-17 cytokine family, up to now, the main focus was given to IL-17A and IL-17A/F in BP. In this study, our results promote the investigation of IL-17 RA and IL$17 \mathrm{RC}$ as new relevant predicting factors in BP. Furthermore, we previously showed that IL-17A/F was a relevant marker of BP outcome (26), and a recent publication showed promising results in the use of anti-IL-17A by using both animal model of BP and a human-derived model of BP (27). Nevertheless, the therapeutic approach targeting the IL-17 axis should be seen from a broader angle. Actually, the IL-17RA isoform is ubiquitous and can combine with most of the other IL-17 receptor isoforms to conduct the message delivered by the different IL-17 isoforms. Indeed, we showed an increase of IL-17RA and IL-17RC at time of diagnosis of patients with BP. Thus, blocking the IL-17RA isoform would limit the effects of IL-17A and IL-17F. Although this still needs to be demonstrated, this could also limit the effects of other IL-17 isoforms that require the IL-17RA to transduce their effects such as the IL-17E, whose concentration is increased in the BF of BP patients. In contrast, this could favor the antiinflammatory effects of IL-17B, which is independent of IL-17RA. Therefore, in BP like in other dermatologic diseases such as psoriasis, inhibiting IL-17RA may represent a good therapeutic option (48-50).

Taken together, the results of our study provide a better understanding of the pattern of IL-17 and IL-17 receptor isoforms in BP. In particular, this is the first study investigating the expression of IL-17 receptors by immune cells from patients with BP. We showed that, in BP, the expression of IL-17RA and IL-17RC in monocyte and macrophages are related to disease outcome, whereas IL-17B was negatively correlated to disease activity. Although further studies are required to demonstrate the

\section{REFERENCES}

1. Joly P, Baricault S, Sparsa A, Bernard P, Bédane C, Duvert-Lehembre S, et al. Incidence and mortality of bullous pemphigoid in France. J Investig Dermatol. (2012) 132:1998-2004. doi: 10.1038/jid.2012.35

2. Bernard P, Vaillant L, Labeille B, Bedane C, Arbeille B, Denoeux J-P, et al. Incidence and distribution of subepidermal autoimmune bullous skin diseases in three French regions. Arch Dermatol. (1995) 131:48-52. doi: 10.1001/archderm.131.1.48

3. Bernard P, Antonicelli F. Bullous pemphigoid: a review of its diagnosis, associations and treatment. Am J Clin Dermatol. (2017) 18:513-28. doi: 10.1007/s40257-017-0264-2

4. Witte M, Zillikens D, Schmidt E. Diagnosis of autoimmune blistering diseases. Front Med. (2018) 5:296. doi: 10.3389/fmed.2018.00296

5. Kridin K, Ludwig RJ. The growing incidence of bullous pemphigoid: overview and potential explanations. Front Med. (2018) 5:220. doi: $10.3389 /$ fmed.2018.00220 role of the IL-17B/IL-17RB axis in the pathophysiology of this disease, our study highlighted an intricate IL-17 network with potential antagonist effects of these members of the IL-17 family.

\section{DATA AVAILABILITY}

All datasets generated for this study are included in the manuscript/supplementary files.

\section{ETHICS STATEMENT}

The studies involving human participants were reviewed and approved by Ethic Committee of the University Hospital of Reims (CNIL authorization DR-2013-320). The patients/participants provided their written informed consent to participate in this study.

\section{AUTHOR CONTRIBUTIONS}

$\mathrm{PB}, \mathrm{FA}$, and SL designed the study. SN, CM, and SL performed experiments. SN, CM, RL, PB, FA, and SL analyzed clinical and biological data. SN and SL performed the statistical analyses. $\mathrm{CM}, \mathrm{MV}$, and PB contributed to the clinical data collection. SN, $\mathrm{RL}, \mathrm{PB}, \mathrm{FA}$, and SL wrote the manuscript. All authors critically evaluated the data and approved the final version for publication.

\section{FUNDING}

This work was funded by the University of Reims ChampagneArdenne, the French Department of Health's Projet Hospitalier de Recherche Clinique (PHRC) Interrégional 2012 and the association Association pour la Recherche en Dermatologie.

\section{ACKNOWLEDGMENTS}

We thank Dr. Christine Terryn from the PICT platform of the University of Reims Champagne-Ardenne for providing microscopy equipment and for technical assistance. We also thank Dr. Anne Durlach from the laboratory of Pol Bouin for providing all of the histological samples.
6. Genovese G, Di Zenzo G, Cozzani E, Berti E, Cugno M, Marzano AV. New insights into the pathogenesis of bullous pemphigoid: 2019 Update. Front Immunol. (2019) 10:1506. doi: 10.3389/fimmu.2019.01506

7. Murrell DF, Daniel BS, Joly P, Borradori L, Amagai M, Hashimoto $\mathrm{T}$, et al. Definitions and outcome measures for bullous pemphigoid: recommendations by an international panel of experts. J Am Acad Dermatol. (2012) 66:479-85. doi: 10.1016/j.jaad.2011.06.032

8. Stanley JR, Tanaka T, Mueller S, Klaus-Kovtun V, Roop D. Isolation of complementary DNA for bullous pemphigoid antigen by use of patients' autoantibodies. J Clin Invest. (1988) 82:1864-70. doi: 10.1172/JCI113803

9. Giudice GJ, Emery DJ, Diaz LA. Cloning and primary structural analysis of the bullous pemphigoid autoantigen BP180. J Investig Dermatol. (1992) 99:243-50. doi: 10.1111/1523-1747.ep12616580

10. Zillikens D, Rose PA, Balding SD, Liu Z, Olague-Marchan M, Diaz LA, et al. Tight clustering of extracellular BP180 epitopes recognized by bullous pemphigoid autoantibodies. J Investig Dermatol. (1997) 109:573-9. doi: 10.1111/1523-1747.ep12337492 
11. Thoma-Uszynski S, Uter W, Schwietzke S, Schuler G, Borradori L, Hertl M. Autoreactive $\mathrm{T}$ and $\mathrm{B}$ cells from bullous pemphigoid (BP) patients recognize epitopes clustered in distinct regions of BP180 and BP230. J Immunol. (2006) 176:2015-23. doi: 10.4049/jimmunol.176.3.2015

12. Di Zenzo G, Thoma-Uszynski S, Fontao L, Calabresi V, Hofmann SC, Hellmark T, et al. Multicenter prospective study of the humoral autoimmune response in bullous pemphigoid. Clin Immunol. (2008) 128:41526. doi: 10.1016/j.clim.2008.04.012

13. Koga $\mathrm{H}$, Teye $\mathrm{K}$, Ishii $\mathrm{N}$, Ohata $\mathrm{C}$, Nakama $\mathrm{T}$. High index values of enzyme-linked immunosorbent assay for BP180 at baseline predict relapse in patients with bullous pemphigoid. Front Med. (2018) 5:139. doi: 10.3389/fmed.2018.00139

14. Niimi Y, Pawankar R, Kawana S. Increased expression of matrix metalloproteinase-2, matrix metalloproteinase- 9 and matrix metalloproteinase-13 in lesional skin of bullous pemphigoid. Int Arch Allergy Immunol. (2006) 139:104-13. doi: 10.1159/0000 90385

15. Verraes S, Hornebeck W, Bernard P, Polette M, Borradori L. Respective contribution of neutrophil elastase and matrix metalloproteinase 9 in the degradation of BP180 (type XVII collagen) in Human Bullous Pemphigoid. J Investig Dermatol. (2001) 117:1091-6. doi: 10.1046/j.0022-202x.2001. 01521.x

16. Hertl M. T cell control in autoimmune bullous skin disorders. J Clin Investig. (2006) 116:1159-66. doi: 10.1172/JCI28547

17. Fairley JA, Burnett CT, Fu C-L, Larson DL, Fleming MG, Giudice GJ. A Pathogenic role for $\operatorname{IgE}$ in autoimmunity: bullous pemphigoid $\operatorname{IgE}$ reproduces the early phase of lesion development in human skin grafted to nu/nu mice. J Investig Dermatol. (2007) 127:2605-11. doi: 10.1038/sj.jid.57 00958

18. Liu Z, Diaz LA, Troy JL, Taylor AF, Emery DJ, Fairley JA, et al. A passive transfer model of the organ-specific autoimmune disease, bullous pemphigoid, using antibodies generated against the hemidesmosomal antigen, BP180. J Clin Invest. (1993) 92:2480-8. doi: 10.1172/JCI1 16856

19. Nishie W, Sawamura D, Goto M, Ito K, Shibaki A, McMillan JR, et al. Humanization of autoantigen. Nat Med. (2007) 13:378-83. doi: $10.1038 / \mathrm{nm} 1496$

20. Schulze FS, Beckmann T, Nimmerjahn F, Ishiko A, Collin M, Köhl J, et al. Fc $\gamma$ receptors III and IV mediate tissue destruction in a novel adult mouse model of bullous pemphigoid. Am J Pathol. (2014) 184:2185-96. doi: 10.1016/j.ajpath.2014.05.007

21. Hirose M, Recke A, Beckmann T, Shimizu A, Ishiko A, Bieber K, et al. Repetitive immunization breaks tolerance to type XVII collagen and leads to bullous pemphigoid in mice. J Immunol. (2011) 187:1176-83. doi: 10.4049/jimmunol.1100596

22. Liu Z, Giudice GJ, Swartz SJ, Fairley JA, Till GO, Troy JL, et al. The role of complement in experimental bullous pemphigoid. J Clin Invest. (1995) 95:1539-44. doi: 10.1172/JCI117826

23. Natsuga K, Nishie W, Shinkuma S, Ujiie H, Nishimura M, Sawamura D, et al. Antibodies to pathogenic epitopes on type XVII collagen cause skin fragility in a complement-dependent and -independent manner. J Immunol. (2012) 188:5792-9. doi: 10.4049/jimmunol.1003402

24. Fichel F, Barbe C, Joly P, Bedane C, Vabres P, Truchetet F, et al. Clinical and immunologic factors associated with bullous pemphigoid relapse during the first year of treatment: a multicenter, prospective study. JAMA Dermatol. (2014) 150:25-33. doi: 10.1001/jamadermatol.2013.5757

25. Le Jan S, Plée J, Vallerand D, Dupont A, Delanez E, Durlach A, et al. Innate immune cell-produced IL-17 sustains inflammation in bullous pemphigoid. $J$ Invest Dermatol. (2014) 134:2908-17. doi: 10.1038/jid.2014.263

26. Plée J, Le Jan S, Giustiniani J, Barbe C, Joly P, Bedane C, et al. Integrating longitudinal serum IL-17 and IL-23 follow-up, along with autoantibodies variation, contributes to predict bullous pemphigoid outcome. Sci Rep. (2015) 5:18001. doi: 10.1038/srep18001

27. Chakievska L, Holtsche MM, Künstner A, Goletz S, Petersen B-S, Thaci $\mathrm{D}$, et al. IL-17A is functionally relevant and a potential therapeutic target in bullous pemphigoid. J Autoimmun. (2019) 96:104-12. doi: 10.1016/j.jaut.2018.09.003
28. Ramirez-Carrozzi V, Sambandam A, Luis E, Lin Z, Jeet S, Lesch J, et al. IL$17 \mathrm{C}$ regulates the innate immune function of epithelial cells in an autocrine manner. Nat Immunol. (2011) 12:1159-66. doi: 10.1038/ni.2156

29. Moseley TA, Haudenschild DR, Rose L, Reddi AH. Interleukin-17 family and IL-17 receptors. Cytokine Growth Factor Rev. (2003) 14:155-74. doi: 10.1016/S1359-6101(03)00002-9

30. Silva WA, Covas DT, Panepucci RA, Proto-Siqueira R, Siufi JLC, Zanette DL, et al. The profile of gene expression of human marrow mesenchymal stem cells. Stem Cells. (2003) 21:661-9. doi: 10.1634/stemcells.21-6-661

31. Kuestner RE, Taft DW, Haran A, Brandt CS, Brender T, Lum $\mathrm{K}$, et al. Identification of the IL-17 receptor related molecule IL$17 \mathrm{RC}$ as the receptor for IL-17F. J Immunol. (2007) 179:5462-73. doi: 10.4049/jimmunol.179.8.5462

32. Wright JF, Guo Y, Quazi A, Luxenberg DP, Bennett F, Ross JF, et al. Identification of an interleukin $17 \mathrm{~F} / 17 \mathrm{~A}$ heterodimer in activated human CD4+ T cells. J Biol Chem. (2007) 282:13447-55. doi: 10.1074/jbc.M700499200

33. Wright JF, Bennett F, Li B, Brooks J, Luxenberg DP, Whitters MJ, et al. The human IL-17F/IL-17A heterodimeric cytokine signals through the IL-17RA/IL-17RC receptor complex. J Immunol. (2008) 181:2799-805. doi: 10.4049/jimmunol.181.4.2799

34. Gaffen SL. Structure and signalling in the IL-17 receptor superfamily. Nat Rev Immunol. (2009) 9:556. doi: 10.1038/nri2586

35. Lee J, Ho W-H, Maruoka M, Corpuz RT, Baldwin DT, Foster JS, et al. IL-17E, a novel proinflammatory ligand for the IL-17 receptor homolog IL-17Rh1. J Biol Chem. (2001) 276:1660-4. doi: 10.1074/jbc.M0082 89200

36. Maezawa $Y$, Nakajima $H$, Suzuki K, Tamachi $T$, Ikeda K, Inoue J$\mathrm{I}$, et al. Involvement of TNF receptor-associated factor 6 in IL-25 receptor signaling. JImmunol. (2006) 176:1013-8. doi: 10.4049/jimmunol.176. 2.1013

37. Fallon PG, Ballantyne SJ, Mangan NE, Barlow JL, Dasvarma A, Hewett DR, et al. Identification of an interleukin (IL)-25-dependent cell population that provides IL-4, IL-5, and IL-13 at the onset of helminth expulsion. J Exp Med. (2006) 203:1105-16. doi: 10.1084/jem.20051615

38. Chang SH, Reynolds JM, Pappu BP, Chen G, Martinez GJ, Dong C. Interleukin-17C promotes Th17 cell responses and autoimmune disease via interleukin-17 receptor E. Immunity. (2011) 35:611-21. doi: 10.1016/j.immuni.2011.09.010

39. Shi Y, Ullrich SJ, Zhang J, Connolly K, Grzegorzewski KJ, Barber MC, et al. A novel cytokine receptor-ligand pair: identification, molecular characterization, and in vivo immunomodulatory activity. J Biol Chem. (2000) 275:19167-76. doi: 10.1074/jbc.M910228199

40. Vaillant L. Evaluation of clinical criteria for diagnosis of bullous pemphigoid. Arch Dermatol. (1998) 134:1075. doi: 10.1001/archderm.134.9.1075

41. Lévy-Sitbon C, Barbe C, Plee J, Goeldel A-L, Antonicelli F, Reguiaï $\mathrm{Z}$, et al. Assessment of bullous pemphigoid disease area index during treatment: a prospective study of 30 patients. Dermatology. (2014) 229:116-22. doi: 10.1159/000362717

42. Riani M, Le Jan S, Plée J, Durlach A, Le Naour R, Haegeman G, et al. Bullous pemphigoid outcome is associated with CXCL10-induced matrix metalloproteinase 9 secretion from monocytes and neutrophils but not lymphocytes. J Allergy Clin Immunol. (2017) 139:863-72.e3. doi: 10.1016/j.jaci.2016.08.012

43. Giusti D, Bini E, Terryn C, Didier K, Le Jan S, Gatouillat G, et al. NET formation in bullous pemphigoid patients with relapse is modulated by IL-17 and IL-23 interplay. Front Immunol. (2019) 10:701. doi: 10.3389/fimmu.2019.00701

44. Yamaguchi Y, Fujio K, Shoda H, Okamoto A, Tsuno NH, Takahashi K, et al. IL-17B and IL-17C are associated with TNF-production and contribute to the exacerbation of inflammatory arthritis. J Immunol. (2007) 179:7128-36. doi: 10.4049/jimmunol.179.10.7128

45. Dong C. Regulation and pro-inflammatory function of interleukin-17 family cytokines. Immunol Rev. (2008) 226:80-6. doi: 10.1111/j.1600-065X.2008.00709.x

46. Reynolds JM, Lee Y-H, Shi Y, Wang X, Angkasekwinai P, Nallaparaju $\mathrm{KC}$, et al. Interleukin-17B antagonizes interleukin-25-mediated mucosal 
inflammation. Immunity. (2015) 42:692-703. doi: 10.1016/j.immuni.2015. 03.008

47. Clapé A, Muller C, Gatouillat G, Le Jan S, Barbe C, Pham B-N, et al. Mucosal involvement in bullous pemphigoid is mostly associated with disease severity and to absence of anti-BP230 autoantibody. Front Immunol. (2018) 9:479. doi: 10.3389/fimmu.2018.00479

48. Leonardi C, Matheson R, Zachariae C, Cameron G, Li L, Edson-Heredia E, et al. Anti-interleukin-17 monoclonal antibody ixekizumab in chronic plaque psoriasis. N Engl J Med. (2012) 366:1190-9. doi: 10.1056/NEJMoa1 109997

49. Griffiths CEM, Reich K, Lebwohl M, van de Kerkhof P, Paul C, Menter A, et al. Comparison of ixekizumab with etanercept or placebo in moderate-to-severe psoriasis (UNCOVER-2 and UNCOVER-3): results from two phase 3 randomised trials. Lancet. (2015) 386:541-51. doi: 10.1016/S0140-6736(15)60125-8
50. Papp KA, Leonardi C, Menter A, Ortonne J-P, Krueger JG, Kricorian G, et al. Brodalumab, an anti-interleukin-17-receptor antibody for psoriasis. (2012) 366:1181-9. doi: 10.1056/NEJMoa1109017

Conflict of Interest Statement: The authors declare that the research was conducted in the absence of any commercial or financial relationships that could be construed as a potential conflict of interest.

Copyright (C) 2019 Nesmond, Muller, Le Naour, Viguier, Bernard, Antonicelli and Le Jan. This is an open-access article distributed under the terms of the Creative Commons Attribution License (CC BY). The use, distribution or reproduction in other forums is permitted, provided the original author(s) and the copyright owner(s) are credited and that the original publication in this journal is cited, in accordance with accepted academic practice. No use, distribution or reproduction is permitted which does not comply with these terms. 